THE

\title{
MORBID ANATOMY AND PATHOLOGY
}

\author{
OF \\ ENCYSTED AND INFANTILE HERNIA.'
}

BY

C. B. LOCKWOOD, F.R.C.S.,

DEMONGTRATOR OF ANATONY AND OPERATIVE SURGERT AT ST. BARTHOLOMEW'S HOSPITAI ; SURGEON TO THE GREAT NORTHERN CENTRAI HOSPITAI.

Received January 22nd-Read May 25th, 1886.

Considerable surgical importance may be claimed for anything which pertains to the subject of hernia. Hardly any affection is so common or more frequently demands surgical interference, and the simplest case may, when operated upon, present the most disconcerting peculiarities. It might be urged that some of these are so rare as not to be of practical importance, but if such an argument as this possesses any weight it would not apply to encysted hernia.

Every writer upon general surgery describes this variety of the disease, and testifies how interesting it is to all who are engaged in the practical duties of their profession.

The history of this affection is by no means difficult

1 The terms "encysted" and "infantile" are in the following pages considered to indicate a purely anatomical condition. 
to trace, for unlike that of congenital hernia it has never been the subject of any dispute. Sir William Lawrence says $^{1}$ that it was first described by Hey, who met with an example of it in 1764. Sir Astley Cooper, ${ }^{2}$ in his magnificent work, alludes to Hey's observations and depicts what may be considered to be a typical specimen.

Writing in 1838 Sir William Lawrence does not allude to any other observations except these, and the knowledge of this author was so profound that it may be assumed that none other existed. Chelius, ${ }^{3}$ and it may be said South, ${ }^{3}$ writing in 1847, merely refer to the authors which have been mentioned, and make no addition to the subject, and the same may be said of Meckel, ${ }^{4}$ whom they quote. Since that time, although a diligent search has been made, I am unable to ascertain that any fresh knowledge has been gained. ${ }^{5}$ Recent authors may have made here and there new statements, but not such as will bear strict investigation. Indeed, a critical examination of the most authoritative accounts of the anatomy and pathology of encysted hernia reveals many discrepancies and leaves much to be explained. In order to justify this assertion, and because their statements are often misrepresented, it may be best to note, as briefly as is consistent with exactitude, the views which the most eminent writers have formulated, and at the same time an adequate idea may be formed as to what is usually meant by the term "encysted hernia." Fortunately this, so far as authorities are concerned, is not a very formidable undertaking. It may be deemed sufficient if $I$ mention what Hey and

1 Lawrence, Sir William, 'A Treatise on Ruptures,' 5th ed., 1838, sec. 2, p. 576.

2 Cooper, Sir Astley, "The Anatomy and Surgical Treatment of Abdominal Hernia,' 2nd ed., 1827, p. 74, pl. xi, fig. 1.

3 'Chelius' System of Surgery,' South, vol. ii, p, 59, 1847.

4 Meckel, 'Handbuch der 'pathologischen Anatomie,' vol. xi, pl. 1, pp. 379 and 380, Leipzig, 1816.

3 This applies to the writings of Vidal, 'Traité de Pathologie Externe,' tome iv, 1861, and to those of Th. Kocher, 'Handbuch der Kinderkrankheiten;" Tubingen, 1880, "Articles on Hernia;" i, 747, et seq. 
Cooper have said and then refer to more recent writers. The case which Mr. Hey ${ }^{1}$ met with, and to which he gave the name " infantile hernia," was that of a child fifteen months old, and after remarking that the cæcum and beginning of the ilium were contained in the hernia, this author proceeds to say, "I found that the tunica vaginalis was continued up to the abdominal ring, and inclosed the hernial sac; adhering to that sac, by a loose cellular substance, from the ring to within half of an inch of its inferior extremity. . . . The interior or true hernial sac was a production of the peritoneum as usual, and contained only the cæcum or head of the colon. . . . Having removed the proper hernial sac I examined the posterior part of the exterior sac; and found it connected with the spermatic vessels in the same manner as the tunica vaginalis is, when the testis has descended into the scrotum." Everything that this eminent surgeon says about the tunica vaginalis in this description is quite clear and precise, but as regards the true hernial sac his remarks are, so far perhaps, slightly wanting in precision. For instance, it is not said whether its walls were constructed of one or more than one layers of peritoneum, and yet it will be seen presently that this is a most important question. However, Hey explains the pathology of the disease in the following way : "In the fœtus a process of the peritoneum is brought down, through the ring of the external oblique muscle of the abdomen, by the testicle as it descends into the scrotum; which process forms an oblong bag communicating with the cavity of the abdomen, by an aperture in its upper part. This aperture is entirely closed at, or soon after, birth. The upper part of the bag then gradually contracts itself, till the communication between that portion of it which includes the superior and greater part of the spermatic chord, and the lower part of the bag, which includes the testicle and a small share of the chord, is obliterated. The lower part of the process

1 Hey, ' Practical Observations on Surgery,' 3rd ed., 1814. An account of an uncommon species of scrotal hernia, p. 226, et seq. ${ }^{2}$ Ibid., pp. 228 and 229.

VOL. LXIX. 
or bag retains its membranous appearance, and is called tunica vaginalis testis propria ; while the upper part covers an irregular cellular substance, without any sensible cavity, diffused amongst the spermatic vessels, and connecting them together.

"In the hernia which I am describing, the intestine was protruded after the aperture in the abdomen was closed; and therefore the peritoneum was carried down along with the intestine, and formed the hernial sac. It is evident, also, that the hernia must have been produced while the original tunica vaginalis remained in the form of a bag as high as the abdominal ring: on which account that tunic would receive the hernial sac with its included intestine; and permit the sac to come into contact with the testicle. The proper hernial sac, remaining constantly in its prolapsed state, contracted an adhesion to the original process of the peritoneum which surrounded it, except at its inferior extremity : there the external surface of the hernial sac was smooth and shining, as the interior surface of the tunica vaginalis is in its natural state."

Before making any comments upon this very clear statement perhaps it will be best to recount the views of another writer whose name has been prominently associated with this subject. ${ }^{1}$

Sir Astley Cooper, ${ }^{2}$ describing what he terms an encysted hernia, says: "On opening the tunica vaginalis, instead of the intestine being found lying in contact with the testicle, a second bag or sac is seen inclosed in the tunica vaginalis, and enveloping the intestine. This bag is attached to the orifice of the tunica vaginalis, and descends from thence into its cavity ; it generally contracts a few adhesions to the tunica vaginalis, while its interior bears the character of a common hernial sac.

1 Mr. Birkett, article on “ Hernia" in 'Holmes's System,' 3rd ed., vol. ii, 1883, p. 807, \&c., says that "Infantile hernia of Hey and encysted hernia of the tunica vaginalis of Astley Cooper are synonymous terms" (see also Mr. Wood's remarks at p. 485).

2 Cooper, 'Anatomy and Surgical Treatment of Abdominal Hernia,' pt. 1, 2nd edit., 1827, p. 79 . 
"The idea which I have formed of the nature of this variety of hernia is, that the tunica vaginalis, after the descent of the testis, becomes closed opposite the abdominal ring, but remains open above and below it. The intestine descends into the upper part, and elongates both the adhesion and tunica vaginalis, so as to form it into a bag, which descending into the tunica vaginalis below the adhesion, and becoming narrow at its neck, though wide at its fundus, receives a portion of the intestine, which in the following case was too large either to be returned into the abdomen, or to retain its functions whilst it continued in the sac."

The cases which Sir Astley quotes were met with by his colleagues in patients upon whom they operated, but only one of these was verified by a post-mortem examination. It will not be necessary to repeat Forster's description, which Sir Astley quotes. He concluded it by saying that after he had opened the tunica vaginalis, and turned back its edges, there was" "exposed a hernial sac pendent from the ring, and descending towards the testicle." In addition Sir Astley Cooper remarks that two other encysted herniæ were met with at Guy's about that time, one during an operation, the other during dissection. It seems by no means improbable that the latter is the actual specimen which he described and depicted in his great work, and which is still to be found in the museum of Guy's Hospital."

If we compare what Hey and Cooper said, it will be allowed that their views are not dissimilar. They both agree in stating that the tunica vaginalis, in the case of encysted hernia, becomes closed at its upper part, and they both attribute the formation of the hernial sac to intestinal protrusion, but neither of them makes an explicit statement concerning the composition of the hernial sac, whether it consisted of one layer of serous membrane, or

I Ibid., p. 80.

- Sir William Lawrence says that such a one was placed in the museum by Sir Astley Cooper (see Plate xi, fig. 1, Cooper on " Hernia "). 
of more than one. There is, however, one very important circumstance to which I would draw attention. Hey, in describing the closure of the tunica vaginalis, says nothing whatever about adhesions, but simply states that "it gradually contracts itself." Cooper, it will be remembered, says, "The tunica vaginalis, after the descent of the testis, becomes closed opposite the abdominal ring, but remains open above and below." Nothing so far has been said about adhesions, but in the next sentence he remarks, "The intestine descends into the upper part (i. e. of the tunica vaginalis), and elongates both the adhesion and tunica vaginalis, so as to form it into a bag, which, descending into the tunica vaginalis below the adhesion, \&c." Although this account may not be free from ambiguity, yet it implies that adhesions closed the tunica vaginalis, and that they actually entered into the formation of the hernial sac. Without assuming that this interpretation of Sir Astley Cooper's statement is correct, I will proceed to quote what has been written by authorities who have succeeded him, but before doing so it is significant to observe that Sir William Lawrence neither refers to, nor passes any opinion upon, Sir Astley Cooper's statement. Mr. Birkett, ${ }^{1}$ moreover, simply says that the ventral orifice of the processus vaginalis becomes closed, "but the canal persisting from that point to the testis. The hernia slowly pushes before it the parietal peritoneum of the abdomen into this sheath, and when the parts are dissected it is seen that the tunica vaginalis is continued up to the abdominal ring, and encloses the hernial sac, as Mr. Hey describes." Mr. Birkett, it is superfluous to point out, does not mention adhesions in connection with the true hernial sac, and merely remarks incidently that it is made of serous membrane; he does not give a detailed account of its structure.

Although at the risk of wearying the reader by constant repetition, yet since it is conducive to a clear conception

1 Birkett, ' Holmes's System of Surgery,' 3rd ed., 1883, p. 807, et seq., vol. ii. 
I would venture to quote more authorities upon this subject.

Writing in the present year (1885), Mr. John Wood ${ }^{1}$ says as follows: "The canal of Nuck [processus vaginalis testis] becomes closed first at the deep ring, leaving a cicatrix which is always more or less traceable. The obliteration extends down the cord to within half an inch of the testicle. The serous membrane degenerates and is transformed into connective tissue, which more firmly binds together the elements of the cord. Sometimes the obliteration extends only to the parts near the deep ring. Then, while the cicatrix is still weak, some violent crying or coughing efforts of the child protrude the bowel, pushing and dilating the cicatrix before it, and a fresh sac of peritoneum is invaginated from above into the upper part of the large tunica vaginalis, which is pushed before it into the scrotum. We have thus formed that kind of children's rupture with a double sac which is called infantile [or encysted] hernia, fig. 1130 (v. Fig. 1, p. 486). In this there are three layers of serous membrane placed in front of the bowel in the scrotum, viz. two layers of the invaginated tunica vaginalis, and one of the fresh, or real sac of the hernia.

The expression "pushing and dilating the cicatrix before it," which Mr. Wood uses in describing the way in which the extruded bowel forms the hernial sac, certainly leaves an impression upon the mind that the hernial sac may be formed, in part at least, of cicatricial tissue. The very clear figure (Fig. 1, p. 486) which accompanies the description would seem to show that the sac which contained the hernia consists of two layers of serous membrane, and that the original communications between the tunica vaginalis and the peritoneal cavity had become entirely obliterated, in truth, it answers perfectly to the graphic description of Forster" "A hernial sac

1 Article on “ Hernia," Ashhurst's 'Encyclopædia,' vol. v, p. 1132, fig. 1130, 1885. I am indebted to Mr. Wood for permission to reproduce this diagram (v. Fig. 1, p, 486).

2 Cooper, loc. cit., p. 79, et seq. 
Fig. 1.

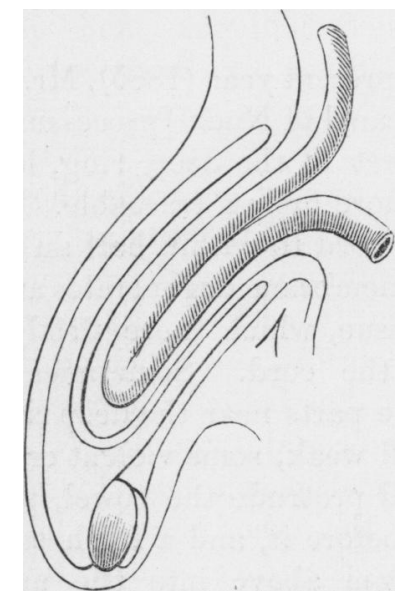

Diagram of infantile (or encysted) hernia. Copied from fig. 1130. Ashhurst, vol. $\mathbf{v}$ (Wood).

pendent from the ring, and descending towards the testicle."

It can hardly be denied that a perusal of these various quotations leaves an impression that the authors of them seem to imply that, in some way or other, cicatricial tissue enters into the composition of the sac of an encysted hernia, but should any doubt remain upon this point it may be dissipated by referring to the writings of Mr. Timothy Holmes. Speaking of this variety of hernia this author says," "This may occur in consequence of adhesions having obstructed the neck of the infundibuliform process and formed a membrane. This membrane being distended by the protruding bowel, forms a hernial sac for it."

Leaving aside for a moment the question of the cicatrix, it cannot be doubted that Mr. Wood and Mr. Holmes describe and delineate that which most surgeons would

1 'A Treatise on Surgery,' T. Holmes, 1882, p. 647, fig. 312. The diagrams which Mr. Holmes gives are, by his kind permission, introduced in Figs. 2 und $3, \mathrm{pp} .488$ and 492. 
consider a representative encysted hernia. ${ }^{1}$ However this may be, Mr. Erichsen depicts and describes quite a different variety. Since Mr. Erichsen's account is a very brief one perhaps it may be given. "Encysted hernia of the tunica vaginalis, or infantile hernia, as it has been somewhat absurdly termed, occurs in those cases in which the funicular portion of the tunica vaginalis is partly obstructed by a septum, or by being converted into filamentous tissue, but in such a way as to leave a pouch above, which is protruded down behind or into the tunica vaginalis, so that it lies behind the cavity."

The last sentence certainly admits two alternatives; in one event the hernial pouch may bulge into the tunica vaginalis, and in the other simply lie behind it; the latter is probably the case which he depicts. ${ }^{2}$ But, although the words "septum" and "filamentous tissue" are met with in this account, used in connection with the method of closure of the funicular portion of the tunica vaginalis, yet it is not clearly stated what those structures may have to do with the formation of the hernial sac. In any case, judging from the diagram, we have now to deal with a hernia quite different from that which, up to this point, has been referred to, unless it be thought that Mr. Erichsen's account tallies with that which has been quoted from Hey.

A glance at Mr. Erichsen's diagram shows how much it differs from that which has been taken from Mr. Wood's writings. It would not appear necessary to attempt to reconcile these conflicting authorities, for, according to Mr. Timothy Holmes, ${ }^{8}$ they are both correct. This author figures and describes two varieties of encysted hernia; one, already mentioned, like Mr. Wood's, a hernial sac pendant from the ring, the other, like Mr. Erichsen's, a pouch behind the open processus vaginalis.

${ }^{1}$ See also Bryant, 'The Practice of Surgery,' ed. iv, vol. i, 1884, p. 732, fig. 264.

2 Erichsen, 'The Science and Art of Surgery,' vol. ii, ed. 8, p. 816, fig. 797.

3 Loc. cit., p. 647, figs. 311 and 312 (for copies, see Figs. 2 and 3, pp. 488 and 492). 
FIG. 2.

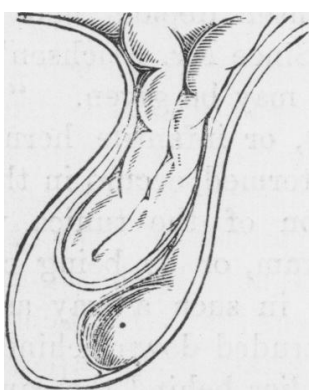

Diagram (copied from Holmes) whose description is as follows:- "Another variety of infantile hernia (the encysted form). The bowel instead of passing behind the closed funicular process has distended the membrane which closes its upper end, and has pushed itself into the funicular process, the upper or back wall of which envelopes it. In this case, therefore, the hernial sac is furnished by the funicular process itself, and only two layers of peritoneum cover the intestine."

There can be little doubt but that Mr. Holmes has expressed the usually accepted views upon this point; and most surgeons and pathologists would concede that there are, in fact, two varieties of encysted hernia. Mr. E. Owen, ${ }^{1}$ who met with an example of the disease, which will be mentioned presently, is of this opinion, and his book upon children's diseases affords very clear diagrams of the two varieties. In order to avoid confusion it will be best to mark each of these varieties of encysted hernia with a definite name. Those which Mr. Holmes uses, although perhaps open to objection, will serve the purpose." In the first place the term "encysted hernia" will be applied to the condition in which, when the unobliterated processus vaginalis is opened, ${ }^{3}$ a hernial sac is seen pendant from the internal ring; and secondly, the term " infantile hernia" will be applied to those cases in which, when the unobliterated processus vaginalis has

1 'The Surgical Diseases of Children,' 1885, p. 345, figs. 57-8.

2 Holmes, p. 647, figs. 311 and 312.

3 The term "processus vaginalis" is applied to the process of peritoneum which accompanies the transition of the testis, and which afterwards becomes tunica vaginalis propria and ruinæ processus vaginalis. 
been opened, a hernial sac or pouch is found behind it, and bulging into it.

Without endeavouring at present to determine to which of these varieties the herniæ described by Hey and Cooper belonged, it may be remarked that most of the authorities who have been mentioned confine themselves, so far as I can judge, to the elucidation of the anatomy and pathology of the encysted form. With regard to the other sort, the infantile, it is true that Mr. Erichsen figures it, but Mr. Holmes throws a certain doubt upon its genuineness, for he says that the diagram which he gives is intended to represent "the assumed condition of the parts in infantile hernia." However, he proceeds to discuss the manner of its formation, and says, "In this form the communication between the peritoneal cavity and the infundibuliform process leading into the tunica vaginalis is obstructed at or about the external (or superficial) ring, but the process itself is not obliterated, so that the cavity of the tunica vaginalis extends up to the external ring. Then a hernia comes down and generally slips behind this upper prolongation of the tunica vaginalis (fig. 311)." (See fig. 3, p. 492.)

This completes a summary of the current views upon the subject of encysted and infantile hernia. With all due deference one cannot help saying that when they are submitted to a critical examination they will be found wanting in scientific precision. In order to support this opinion I will confine myself, for the present, to the pathology of encysted hernia, and without further preliminaries, discuss a question which seems to go to the very root of the matter; and it is this : What has cicatricial tissue to do with the formation of the hernial sac? It cannot be denied that although Hey said nothing whatever about cicatrices, adhesions, or septa, yet we find them mentioned by Sir Astley Cooper and succeeding authors, until at last the greatest importance seems to be attached to them. 
In order to determine this most important question two methods of investigation are open to us : in the first place, to inquire whether the upper part of the processus vaginalis is ever closed by adhesions or cicatrices capable of forming a septum suitable for the creation of a hernial sac ; and next, to see whether the sac has the appearances which it might be expected to possess had it been formed of cicatricial tissue.

With regard to the first part of this inquiry, it seems very hard to discover upon what exact basis of fact the actual existence of the septum, which is assumed to close the processus vaginalis, rests.

I have been unable to discover that any author says that he has actually seen such a thing. Although it is a hopeless task to try and prove a negative, yet it cannot be without influence upon this argument to notice that Wrisberg, ${ }^{1}$ Seiler and others investigated the processus vaginalis with great industry, and that none of them mention such a thing, and it. is hardly in accordance with our general knowledge of tubes with endothelial linings to conceive of their closure by septa. The function of the processus vaginalis is to give passage to the testicle, and when it has done this it not only ceases to grow, but undergoes retrograde atrophic changes. Under the circumstances we are considering, in which it becomes the receptacle for an encysted hernia, the very opposite occurs; the processus vaginalis grows and its lumen increases, a fact which diminishes the likelihood of its occlusion by a septum. It must be confessed that an inspection of Cloquet's drawings ${ }^{2}$ suggests very strongly, whatever normal anatomy may afford or a priori reasoning suggest, that, nevertheless, hernial sacs may be partitioned by septa. From a septum in a hernial sac to one in the processus vaginalis is not a

1 " De testiculorum ex abdomine in scrotum descensu, etc.," 'Comment. Soc. Reg. Scient. Gotting.' 1800, p. 173, et seq. 103 examinations are recorded by Wrisberg, and Mr. Birkett attributes 54 to Camper and 21 to Seiler (art. in 'Holmes's System,' 3rd edit., vol. ii).

2 'Recherches sur les causes et l'anatomie des hernies abdominales,' Paris, 1819. 
long leap. However, an examination of Cloquet's specimens themselves, which are in the Dupuytren Museum, ${ }^{1}$ shows that any partial septa which are present in them are really due to pleats in the walls of the hernial sac, each accompanied by a corresponding constriction upon the exterior, and very like the folds of the large intestines. ${ }^{2}$ It would not be right to draw definite conclusions from these specimens, because they are simply hernial sacs which have been dried and varnished, but they hardly suggest the existence of septa of cicatricial tissue. It is true that they show complete constriction of the hernial sac $;$ but even in this case, I do not think it has ever been argued seriously that an encysted hernia could be produced by an intussusception of one part into another, for, owing to the gradual nature of the constrictions, this would seem an impossibility.

It is not for a moment pretended that the arguments which have just been adduced, prove the impossibility of the processus vaginalis ever being occluded by a cicatricial septum, but it can hardly be denied that they suggest the improbability of such an event. However this may be, under these circumstances it seemed best that they should be stated, for the sequel will show that the pathology of encysted hernia depends more upon the whole weight of evidence than upon any particular fact. The reason for this will be clear when we begin to array the evidence which has been afforded by an examination of the various specimens of encysted hernia which are to be found in the various London museums, and owing to the very great kindness and courtesy of the curators, I have been permitted to dissect and examine them at my leisure. In order to avoid the embarrassment which the multiplication of intricate details sometimes causes perhaps I may be permitted to begin with a general statement of results.

Just for the moment it may be said that the various

1 Cloquet's specimens are Nos. 269 to 315.

2 See Specimens 236 and 306.

3 See Specimens 310, 312, 314, and 315. 
specimens seem to belong to two very distinct types. In both of these it is an essential feature that the sac of the tunica vaginalis should be very large, reaching almost, if not quite, as far as the peritoneum; but the question of its communicating with the cavity of that membrane is a point which will be discussed presently. This much having been premised, it may be stated that the two apparent varieties are those which have been already spoken of as " encysted $\times$ Fig. 3.

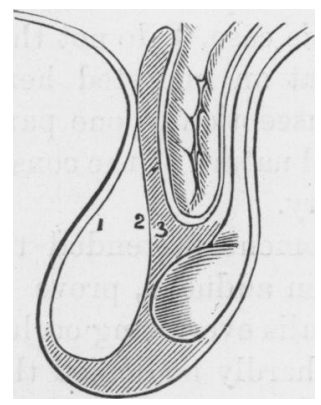

Diagram (copied from Holmes, fig. 311) of the (assumed) condition of the parts in an infantile hernia. The tunica vaginalis (1) is closed above, at or near the external inguinal ring, but its funicular portion is open. The bowel in the hernial sac lies behind this funicular portion, and is represented in the diagram as having made its way between the funicular process and the cord. The relation of the sac to the cord seems, however, to be variable. The bowel is covered in cutting down from the skin by three layers of peritoneum, viz. 1 and 2, the opposite surfaces of the funicular process, and 3 the anterior layer of the peritoneal hernial sac.

hernia " and "infantile hernia." Specimens 2497 and $294.7^{50}$ in the Guy's Hospital Museum (v. Figs. 5 and 6, pp. 495 and 498) and Specimen C. D. 20 in the Museum of St. Mary's Hospital ( $v$. Fig. 8, p. 510) may be considered representative of the encysted. Indeed, there is not much doubt but that one of them, Specimen 249750, Guy's, is the very one which Sir Astley Cooper depicted ; ${ }^{1}$ whilst the St. Mary's specimen was described as an encysted hernia

1 Cooper on “ Hernia," plate xi, fig. 1. 
in the British Medical Journal. ${ }^{1}$ The second variety of encysted hernia which the museums contain is clearly of the sort which has already been described under the name

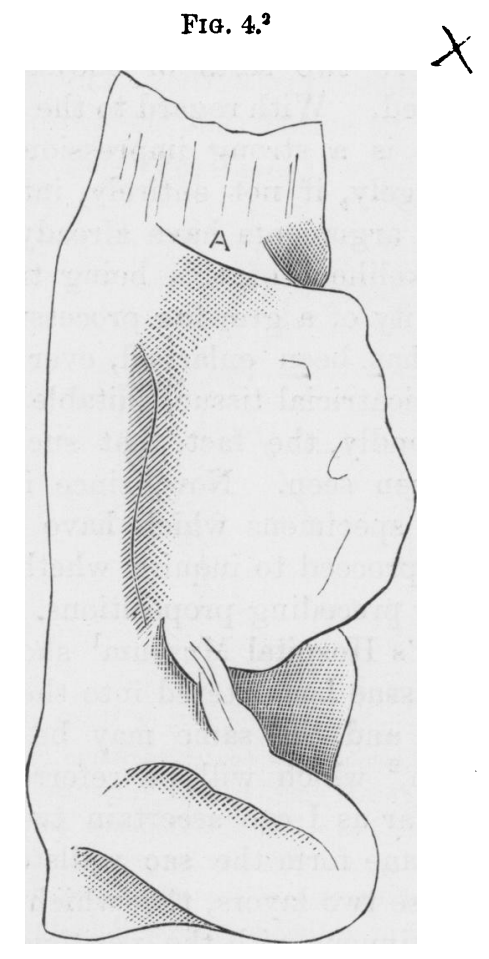

Drawing of an infantile hernia, specimen R. 24, in the St. Thomas's Hospital Museum. The bulging of the hernial sac into the tunica vaginalis is shown and also the fold (plica vascularis), which extends from lower extremity of the sac to the epididymis. In the catalogne the specimen is named, "encysted." A, mouth of sac.

of infantile, and which consists of a pouch or bag of peritoneum pushed down behind the greatly enlarged tunica vaginalis. ${ }^{3} \quad$ Specimens $2488^{50}$ in the Guy's Hospital

1 'British Medical Journal,' Aug. 1, 1874, p. 140, E. Owen.

${ }^{2}$ I am indebted to the kindness of Mr. Shattock for permission to examine and draw this specimen.

3 As Hey describes. 
Museum and Specimen R. 24 in the Museum of St. Thomas's Hospital ( $v$. Fig. 4, p. 493) may be considered representative of this class.

It may be remembered that the current views as to the pathology of these two sorts of encysted hernia have already been stated. With regard to the first, it has been shown that there is a strong impression that cicatricial tissue enters largely, if not entirely, into the formation of its sac. Two arguments have already been advanced to show the unlikelihood of this being true; first, the a priori improbability of a growing processus vaginalis, the lumen of which has been enlarged, ever being occluded by a septum of cicatricial tissue, suitable for becoming a hernial sac; secondly, the fact that such a septum has never actually been seen. Now, since it can hardly be denied that the specimens which have been chosen are typical, we may proceed to inquire whether they confirm or contradict the preceding propositions. The two specimens in the Guy's Hospital Museum ${ }^{1}$ show no indication that cicatricial tissue has entered into the construction of the hernial sac, and the same may be said of the St. Mary's specimen, 2 which will be referred to afterwards at length. So far as I can ascertain two distinct layers of serous membrane form the sac walls of these encysted herniæ. Of these two layers, that which lines the interior of the sac is continuous with the peritoneal cavity, whilst that which covers its exterior is continuous with, and forms part of, the tunica vaginalis. ${ }^{3}$

The real importance of this observation will be clearer after awhile, but for the moment we may pause to meet an argument which readily suggests itself, namely, whether after a time even a septum of cicatricial tissue might not assume the characters of the serous membranes in its neighbourhood.

Figs. 5 and 6, pp. 495 and 498.

2 Fig. 8, p. 510

3 Approximating very closely the condition described by Hey, v. a. pp. 481 and 482. 
There is no proof that such an event takes place under any circumstances, and an examination of the specimens (Figs. 5, 6 and 8) affords no evidence in its support. Not only are the two layers of serous membrane of which the

FIG. 5.1
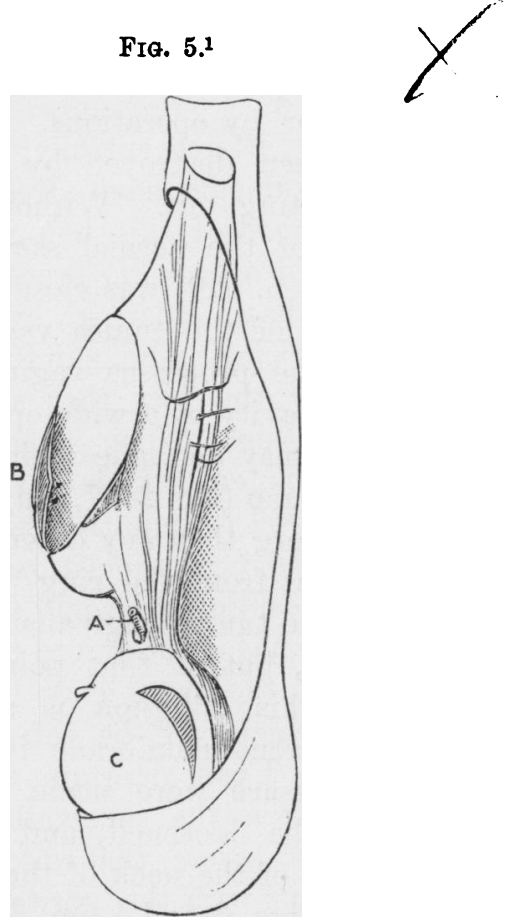

Description of Fig. 5 of "encysted hernia" (v. Catalogue), No. 2497, Guy's Hospital Museum. The two layers which form the sac wall are shown and also the band which passes from the epididymis to its extremity. In the interior of the sac of this hernia, behind, there is a curious pouch made by a transverse fold of serous membrane. B, hernial sac. A, band with spermatic artery in its midst. c, testicle and epididymis.

true hernial sac is composed quite distinct, but there is muscular tissue between them; a point which will be explained later.

1 Dr. Goodhart kindly permitted me to examine and draw this and other specimens. 
If it is clear that the sacs of these encysted herniæ consist not of cicatricial tissue but of a double layer of serous membrane we may now proceed to investigate the crucial question, whether the tunica vaginalis in these cases of encysted hernia communicates with, or has been shut off from, the peritoneal cavity. Allowances must be made, in investigating this, for alterations produced by previous dissection or by operations. The possibility of adhesions having been destroyed by this means is too obvious to need pointing out. Without doubt the front of the upper edge of the hernial sac in the St. Mary's specimen ( $v$. Fig. 8, p. 510) was closely applied, perhaps adherent, to the wall of the tunica vaginalis, but I am of opinion that in it the processus vaginalis communicated with the peritoneal cavity by a wide opening, and I think the same statement may be made with regard to another of these encysted hernim (No. 2497, Guy's), see fig. 5. In a specimen which more than any other might be called a " hernial sac pendant from the ring" (v. Fig. 6, p. 498, Sp. $2497^{50}$, Guy's), the tunica vaginalis is open right up to the neck of the sac, but at that point its walls adhere to one another. This adhesion is so slight and the continuity of the serous membrane is so palpable, that if the smallest pressure were made with a probe the attachments would be loosened, and the specimen as regards the relations of the neck of the sac made like an infantile hernia. (v. Figs. 3 and 4, pp. 492 and 493).

Having now ascertained the condition of the tunica vaginalis in the most typical encysted herniæ, it is unnecessary to say that the opinions which have been quoted concerning the pathology of this disease are unacceptable.

If the various specimens of encysted hernia were diagrammatically represented, it would be seen that they belonged to the infantile type (Fig. 3).

In either case the hernial sac consists of an outer and an inner layer of serous membrane, one formed by a protrusion from the peritoneum, the other by the tunica vaginalis. The differences which are present depend upon the degree 
to which the hernial sac may have bulged into the tunica vaginalis and not to any difference in their actual construction.

Of course this takes for granted that the existence of the infantile variety is admitted and its morbid anatomy acknowledged, but, upon this point, an inspection of the specimens in the various museums leaves absolutely no doubt, and the facts which have been mentioned tend to justify this assertion. The truth of the statement that all the specimens of encysted hernia belong to the sort called infantile, would not be at all obvious if it depended upon a comparison instituted between what may be called exaggerated instances : for example, if an infantile hernia which hardly bulges at all into the tunica vaginalis be compared with one which protrudes excessively (e.g. compare Figs. 4 and 6 ).

But between these extremes intermediate grades exist, and from these a series may be constructed to illustrate the progression from one to the other. Perhaps it is unnecessary at present to do more than mention a specimen of infantile hernia (Sp. R. 24, St. Thomas's Hosp., Fig. 4) which, although typically belonging to the infantile variety, has many of the characters attributed to the so-called encysted.

Before concluding this account of the morbid anatomy of the encysted herniæ, their relation to the posterior wall of the tunica vaginalis may be mentioned. It has been stated that the degree in which the hernial sac protrudes into the tunica vaginalis varies in different specimens, and so far, perhaps, as concerns those which bulge least, nothing requires to be said. However, when the protrusion is considerable, the cyst-like sac is attached to the posterior wall of the tunica vaginalis by a mesentery which extends along the whole length of its posterior surface. This may have been so in Hey's case, although he assumes that the attachment was merely an adhesion formed after the occurrence of the hernia (vide p. 482). It may be added that it is usual to find that the lowest part of this vol. LXIX. 
mesentery attaches the hernial sac to the epididymis, forming a fold (plica vascularis) the importance of which will be explained.

Only one specimen seems to contradict this assertion

Fig. 6.

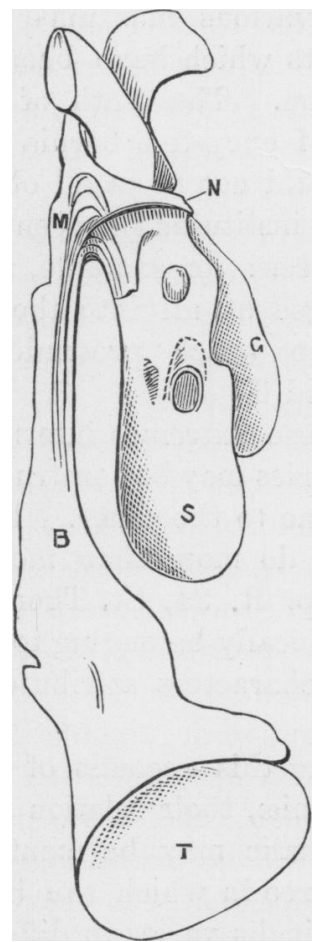

“Encysted Hernia" (v. Catalogue), No. 249750, Guy's Hospital Museum. Showing attachment of sac to the posterior wall of the processus vaginalis; also muscular fibres turning round fornix between the sac and vaginal process. A curious little pouch is seen upon the wall of the hernial sac.

$M$, muscle-fibres; $N$, neck of sac; $G$, contents, gut; B, cord ; 8 , hernial sac; T, testis. (This is probably the specimen delineated by Sir Astley Cooper, Plate xi, fig. 1.)

and it is depicted in Fig. 6, but the difference is more apparent than real, and is due to the extraordinary way in 
which the sac has been protruded into the tunica vaginalis.

That this view is correct will, I think, be clearly shown when the pathology of this affection is discussed.

Having endeavoured to describe the morbid anatomy of the most typical examples of encysted hernia, and having sought to show that they belong to the infantile variety, perhaps it may be as well before advancing any facts concerning their pathology to recapitulate the arguments which have been used to contradict the usual opinions upon the subject.

1. The absence of proof that the processus vaginalis is ever closed by a septum of cicatricial tissue.

2. The improbability of a septum being formed in a processus vaginalis which has presumably grown, and the lumen of which has increased.

3. That the sac of an encysted hernia does not consist of cicatricial tissue, but of two layers of serous membrane.

4. That it is doubtful whether the processus vaginalis is invariably shut off from the peritoneal cavity in these cases, or if it be shut off, the closure is effected in such a way as to exclude the possibility of a septum of cicatricial tissue having existed.

To these destructive arguments may be added the constructive ones which are contained in the descriptions of the various specimens, and as we proceed to discuss their pathology others will be forthcoming.

The various authors who have written upon the pathology of these herniæ have confined their remarks to speculating on the causation of the encysted variety. As far as I am able to judge, the tendency has been to attribute the latter to modifications which take place in cicatricial tissue which is supposed to obstruct the processus vaginalis. Assuming that this "theory" has, in the preceding pages, been disproved, and that it has been substantiated that all the specimens belong, in reality, to the infantile variety, we may now proceed to inquire how infantile hernia is produced. With the exception of Mr. Hey's observations, 
already quoted, and which probably apply to this condition, authorities say but little. Mr. Holmes says that their origin is a hernia which slips behind the upper prolongation of the tunica vaginalis. This is hardly an explanation of the pathology of infantile hernia, and, in the absence of any other, nothing remains but to consult the various specimens for information as to their elucidation. Whatever help clinical history may afford in other cases, in this it is valueless. It is true that Mr. Hey's and Mr. Owen's cases happened in infants, but, as Mr. Birkett points out, infantile hernia may seem to originate for the first time during adult life. Many facts, more particularly the state of the tunica vaginalis, irresistibly suggest that infantile hernia is due to some peculiarity in the process of development. It has been remarked already that it is an essential feature in this disease that the cavity of the tunica vaginalis be of large size and either in communication with the abdomen or separated from it by the apposition and adhesion of its walls opposite the neck of the hernial sac.

Those who are acquainted with hernia into the tunica vaginalis (congenital hernia) will at once perceive that this is a condition with which they are familiar. Without doubt in cases of hernia into the tunica vaginalis the patency of that membrane is the predisposing cause of the rupture, and it must be exceedingly rare, as Kocher points out, for a protrusion to occur early enough to prevent the closure of this funicular process.

However this may be, well authenticated cases of hernia into the tunica vaginalis show that the congenital defect of patency existed long before the rupture, so that, even if it be clearly substantiated that in the case of an infantile hernia, the rupture had not shown itself until adult life, it would not invalidate the assumption that its predisposing cause was a developmental defect. An examination of the specimens of infantile hernia (including the encysted in this term) creates a very strong impression that events connected with the transition of the testicle 
have a predominating influence upon the origin of the disease.

It seems reasonable, therefore, to begin with a review of the various events which are associated with that act, and afterwards inquire whether they throw any light upon this subject.

Few questions have been studied with so much care and diligence as the transition of the testis, and the result has been set forth in a formidable literature. It seems unnecessary in this place to endeavour to reconcile the conflicting statements of various authorities; they have been excellently summarised in the elaborate monograph of Godard. ${ }^{1}$

For the purpose of this inquiry, Mr. Curling's account ${ }^{2}$ of the transition of the testis may be taken as a basis, for it is most in accordance with that which can be seen. As far as it seems possible to investigate this subject by dissection Mr. Curling has succeeded, and unless new methods had been adopted, little would remain to be added to his description. It is not proposed to enter into an elaborate and detailed account of the results which have been obtained by the examination of more than twenty human fœtuses of various sizes. It has been implied that, so far as concerns dissection, they confirm nearly all that $\mathrm{Mr}$. Curling has said. In addition, the question has been studied in the following way, whole fœetuses were placed in a large quantity of a solution of chromic and hydrochloric acid until the soft tissues were hardened and the bones were.decalcified. The whole pelvis was then suitably embedded in paraffin, and a series of thin sections cut with a large microtome.

Having mentioned these particulars, we may now proceed to sketch the result of the various investigations, but, since it is proposed to discuss this subject at greater

1 Godard, "La Monorchidie et la Cryptorchidie chez l'homme," 'Comptes Reudus,' 1856, p. 315.

'A Practical Treatise on the Diseases of the Testicle,' T. B. Curling, 4th ed., 1878, p. 17, et seq. 
length at another time, the narrative will be kept as free as possible from controversy. It will be sufficient for present purposes if the position and attachments of the testicles, as they are usually found at the seventh month of intra-uterine life, be first described.

At this time, as Fig. 7 shows, the testis is situated

FIG. 7.

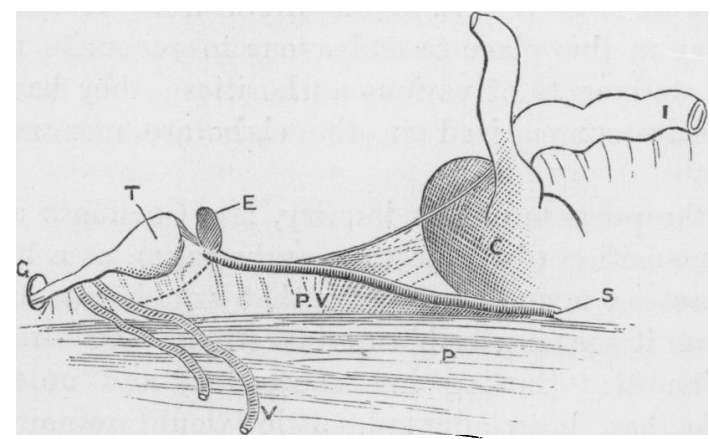

Drawing made from a seven or eight months fœetus to show the fold (plica vascularis) which connects the testis with the cæcum.

T, testicles; r, epididymis ; P, psoas; V, vas deferens; G, plica gubernatrix, disappearing into processus vaginalis; P.V, plica vascularis; C, cæcum; s, spermatic artery; I, ilium.

in the iliac fossa, a little above the internal abdominal ring, and is attached to the front of the psoas muscle by the mesorchium, which is simply a fold of peritoneum about one third of an inch wide. In its free border the body of the testicle and epididymis lie a little way apart, the latter being nearer the attachment. In addition, the mesorchium has two folds which extend upwards and downwards from the testicle. The upper contains the spermatic vessels and a quantity of unstriped muscle-fibres," and may be called the "plica vascularis."

1 All statements made in this paper concerning muscular fibres have been repeatedly verified by inicroscopic examination. 
The muscle ${ }^{1}$ belongs to the gubernaculum testis, and will be fully described hereafter. The upper part of the plica vascularis of the right side, as Wrisberg ${ }^{2}$ states, ends either upon the vermiform appendix, the mesentery, the cæcum, or the ileum. Without doubt the main portion passes to the common mesentery, which, at this period, belongs to the cæcum and ileum, the remainder being subsidiary; on the left side the plica vascularis passes to the sigmoid flexure. The inferior fold of the mesorchium is called the plica gubernatrix, because it contains the testicular end of the gubernaculum testis. In an eight months' fotus the lower end of the plica gubernatrix disappears into the orifice of the processus vaginalis, which has commenced to be formed. The way in which a sort of test-tube of peritoneum accompanies the transition of the testicle is too well known to call for comment, but the manner of its production requires to be described. It seems natural to suppose that the serous membrane accompanies the gland on account of their mutual adhesion. Although this may be an element in the case, another factor must be taken into consideration, for there can be little doubt that the processus vaginalis moves towards the scrotum in advance of the testicle. ${ }^{3}$ As a rule, the peritoneal test-tube does not precede its contents by many lines, but the distance may be so palpable as to preclude the possibility of the testicle having pushed or dragged its serous covering towards the scrotum. A certain degree of support is afforded to these observations by the well-known fact that when the testis is undescended a process of peritoneum may reach towards the scrotum. This is shown in many museum specimens $(e . g$. $2339^{30}, 2339^{50}, 2339^{25}$ in the Guy's Hospital Museum,

1 This may be the fold sometimes named after Seiler, see Banks, "On the Wolffian Body, \&c.,' Edinburgh, 1864, but Sappey calls the whole mesorchium “ Seiler's fold," ' Traité d'Anatomie,' vol. iv, p. 604.

2 Loc. cit., p. 230.

3 Quain's 'Anatomy,' 9th ed., vol. ii, p. 908.

4 Lawrence, p. 569, also Cloquet, p. 23 ('Les Causes,' \&c.). 
also Sp. 91, S. IX in the St. George's Hospital Museum). Since in some of these cases the testicle is adherent in the iliac fossa, it is obvious that it could not have pushed down the peritoneum. If the superior terminations of the gubernaculum be examined, both anatomically and microscopically, the reason why the processus vaginalis mores in advance of the testicle is explicable. The fibres of that muscle are inserted, not only into the epididymis, vas deferens and testicle, but also into the peritoneum. At about the seventh month of intra-uterine life, muscular fibres may be seen inserted into the extremity of the processus vaginalis, and, moreover, many of them are prolonged up the mesorchium into the plica vascularis, and so onwards to the peritoneum which lines the posterior wall of the abdomen. The lower attachments of the gubernaculum are described so clearly by Mr. Curling that a detailed description seems unnecessary. It is generally recognised that it has three main attachments; one to the abdominal wall; another to the pubes, the lower part to the sheath of the rectus and the root of the penis; and a third to the bottom of the scrotum. Repeated dissections substantiate these statements. Perhaps it may be mentioned that some of the fibres of the portion which mingles with the wall of the abdomen pass downwards into Scarpa's triangle and are not unimportant in affording a plausible reason for the occasional passage of the testicle into the thigh.' It is quite unnecessary to say that the function of pulling the testicle into the scrotum is attributed to these divisions of the gubernaculum. The first pulls it as far as the internal abdominal ring, the second to the pubes, and the third deposits it in its final resting place.

If we proceed to consider the various events which accompany the transition of the testicle, I think it will be admitted that the gubernaculum must exert a certain

1 Mr. McCarthy mentions this occurrence, but attributes it to abnormal fibres of the gubernaculum, Quain's 'Dictionary of Medicine,' 1882, p. 1606. 
amount of force. For instance, if a fœtus be chosen in which the gland is about to pass through the abdominal wall, and traction be made upon the gubernaculum, it is clear that as the testicle travels towards the scrotum not only the mesorchium and its contents and the processus vaginalis, but the peritoneum which lines the posterior wall of the abdomen, moves with it. In consequence of this locomotion of the serous membrane, the cæcum and ileum on the right side, and the sigmoid flexure upon the left, attain a lower position in the abdomen, a circumstance upon which both Scarpa ${ }^{1}$ and Wrisberg ${ }^{2}$ have commented. That the transition of the testicle has an important influence upon the movements of the viscera is suggested by the fact that in the cases of retained testicle the cæcum may fail to complete its descent into the iliac fossa ${ }^{3}$. The exact contrary of this may happen, and the cæcum or the ileum be dragged with the testicle into the scrotum, producing a congenital cæcocele. Wrisberg, ${ }^{4}$ Scarpa ${ }^{5}$ and Cloquet ${ }^{6}$ mention such cases and say that the cæcum was attached to the testicle by a fold which they identify as the plica vascularis, but without naming it. I have been so fortunate as to find a congenital cæcocele in a very young infant. In it the plica vascularis had entirely disappeared, but upon the back of the hernial sac there was a quantity of muscular fibres and fibro-areolar tissue, which passed from the back of the testicle upwards to the cæcum. $^{7}$ These bands were parallel to and adjoining the spermatic vessels, and without doubt the hypertrophied representatives of those of the gubernaculum which before

1 'A Treatise on Hernia,' translated by Wishart, Edinburgh, 1814, p. 38.

2 Loc. cit.., p. 230.

${ }^{3}$ See a paper by author, ‘ Brit. Med. Journ.,' Sept., 1882, p. 575, “ Abnormalities of the Cæcum and Colon with Reference to Development."

4 Loc. cit., p. 233.

5 Loc. cit., p. 203.

6 'Causes, \&c., des Hernies,' p. 23. See also Cruveilhier, 'Anatomie Pathologique,' vol. iii, p. 307, Paris, 1849.

7 My friend Mr. D'Arcy Power kindly verified this fact. 
birth normally exist in this situation. It would be illogical to argue that because these were present therefore they were responsible for the abnormal descent of the cæcum, but it is not impossible. This specimen is important in other respects and will be mentioned again. Assuming it is true that a general locomotion of the peritoneum of the back of the lower part of the abdomen accompanies the transition of the testicle, it remains to be decided whether the gubernaculum is capable of such an effort. Judging from the amount of its muscularity this question may be answered in the affirmative, but it is doubtful whether all of its attachments are adequate. It is easily appreciated that the portions which adhere to the abdominal walls and to the pubes may, by their contraction, move onwards the testicle and peritoneum, because they spring from definite fixed points, but the part which arises from the bottom of the scrotum seems entirely deficient in this respect. Doubtless the scrotal fibres influence the ultimate destination of the gland, but properly prepared specimens show that the actual work of transition is performed by a band of fibres which originates in the perinæum. This is exceedingly well displayed in an infant in whom I found a congenital hernia of the cæcum. In this case the perinæum is occupied by a quantity of unstriped muscular tissue, continuous behind, with the external sphincter and tissues over the tuber ischii, whilst in front its fibres mingle with those of the scrotum, and those which have been mentioned as passing up the posterior wall of the hernial sac to the cæcum. It is not impossible that in this case the dissection was facilitated by the muscle being hypertrophied. It is never easy to follow bands of unstriped muscle with the scalpel, and although the fœtal perinæum always contains them in abundance, it would be rash, without the aid of microscopic sections, to make explicit statements concerning them. However, the combined methods show that this portion of the gubernaculum after emerging from the perinæum is attached to the extremity of the processus vaginalis, the testicle, and 
epididymis, and, moreover, that its fibres extend up the posterior surface of the processus vaginalis towards the peritoneum which lines the back of the abdomen. Clearly these are the muscular bands which have been already (p. 502) notified in the plica vascularis.

The preceding statements derive a certain degree of support from the fact that when the testicle exceeds its proper excursion, and passes into the perinæum, it has been seen attached to the tuberosity of the ischium by a band which required division before replacement into the scrotum could be achieved. Both Cloquet ${ }^{1}$ and $\mathrm{Mr}$. McCarthy ${ }^{2}$ mention a case of this sort, and $\mathrm{Mr}$. Treves has informed me of a similar one under his care. It is not impossible that the perineal fibres of the gubernaculum may in a degree persist throughout life, for in an exceedingly well-developed subject the subcutaneous tissue in that region contained large quantities of unstriped muscle-fibres.

With regard to the part of the gubernaculum testis which extends up the back of the processus vaginalis and into the plica vascularis, it is interesting to note that, as Cruveilhier $^{3}$ points out, the spermatic cord contains numerous longitudinal bands of unstriped muscle, which he calls the "internal cremaster." I would identify these as being the upward prolongation of the gubernaculum testis, whose importance in relation to infantile hernia will be shown in what follows. Before discussing this branch of the subject, a last word may be spoken upon the question of the locomotion of the peritoneum.

It may be remembered that it has been repeatedly said that only the serous membrane which clothes the back of the abdomen moves towards the groins. But before this is accepted it is necessary to solve the question why other portions are not involved. The problem seems purely anatomical. The peritoneum which lines the inner surface

1 Loc. cit., p. 24, 5. This case was verified by dissection.

2 Loc. cit., p. 1606.

3 Cruveilhier, ' Traité d'Anatomie,' 1874, vol. ii, p. 381, fig. 253. 
of the transversalis fascia and muscle is, both in the fœtus and in the adult, ${ }^{1}$ so closely attached to those structures that its displacement is practically impossible. In this situation in the fœtus, sub-peritoneal tissue is almost absent, and the serous membrane is evenly distributed and devoid of pleats and folds. The contrary is the case with that which lines the iliac fossæ and back of the abdomen, for in this situation, as John Hunter ${ }^{2}$ points out, its laxity is so great and its connections so loose, that ample folds may easily be seized and dragged in any direction. Histological specimens show that everywhere in the region of the psoas muscle the serous sac is underlaid by a great quantity of the most delicate connective tissue, and that an ample cushion of this is prolonged behind the advancing processus vaginalis into the scrotum. A final reason for the displacement of this particular part of the peritoneum is that the fibres of the gubernaculum are especially distributed to it.

Before endeavouring to apply these anatomical and developmental data to infantile hernia, perhaps the most important may be recapitulated.

A. That the lowest attachments of the gubernaculum are in the perinæum.

B. That the gubernaculum is inserted into, and draws the processus vaginalis into the scrotum.

c. That the gubernaculum is prolonged above the testicle to the peritoneum of the posterior wall of the abdomen, and produces an extensive locomotion of it.

If we now return to inspect the various specimens of infantile hernia which have been mentioned, it is palpable that either a fold of peritoneum, or a well-marked fasciculated band of tissue extends from the upper part of the epididymis to the inferior extremity of the hernial sac.

Cloquet makes a similar observation as regards adults, 'Recherches Anatomiques sur les Hernies de l'Abdomen'’ p. 44.

: O Observations on Certain Parts of the Animal Economy,' by John Hunter, 1786. A description of the situation of the testicle, p. 8 . 
This fold is exceedingly well shown in a specimen of infantile hernia which is in the museum of St. Thomas's Hospital ( $v$. Fig. 4), and, owing to the manner in which it arises at the upper end of the epididymis, there is not the slightest difficulty in recognising it as the remains of the plica vascularis. Under ordinary circumstances that reduplication of serous membrane almost entirely disappears, but an examination of the various specimens of congenital hernia in the London museums shows that it has a very great tendency not only to persist, but to attain considerable size and stretch far up the posterior wall of the sac. This point is clearly shown in one of Camper's plates ${ }^{1}$ and in a specimen of congenital hernia which I obtained from a pig. It is of considerable practical importance because the fold indicates not only the position of the spermatic vessels, but also distinguishes certain adhesions which are found in congenital hernia. The plica vascularis has already been mentioned in connection with cæcocele, and its relation to the disease has been noted. It seems unnecessary to say at length how essential a knowledge of the structure is to the practical surgeon.

In reading accounts of operations upon congenital herniæ one is struck by the frequency with which adhesions of the gut to the back of the sac, and to the testicle, are mentioned, and often the significant remark is added that when the adhesion was severed, the spermatic vessels were divided. ${ }^{2}$ If an opinion may be formed from morbid anatomy specimens this disaster may be avoided by simply ascertaining whether the fold or adhesion is the plica vascularis, and to decide this question it is only necessary to trace the band towards the testicle and observe its relation to the epididymis. After this digression the relation of the plica vascularis to the pathology of infantile

1 Camper, 'Icones Herniarum,' ed. by S. J. Soemmerring,' 1801, Tab. iii, figs. 3 and 4.

${ }^{2}$ E. g., Pott's 'Chirurgical Works,' vol. ii, p. 159, 1779; also Vidal, 'Traité de pathologie Externe,' tome iv, 1861. 
hernia may be resumed. It has been stated that Wrisberg and others consider the fold, which I have ventured to call the plica vascularis, an important factor in the causation of congenital hernia of the cæcum and sigmoid flexure, and

FIG. 8.

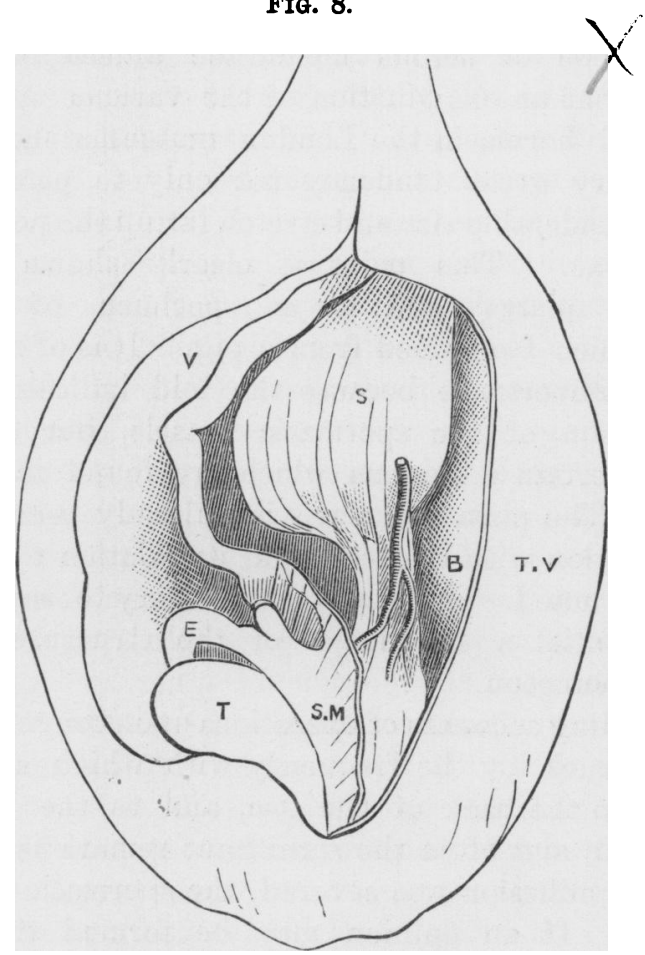

Specimen of "encysted hernia in the Museum of St. Mary's Hospital, Sp. C. D. 20. Shows band passing from epididymis to bottom of sac. The spermatic artery is seen amongst its fibres. The vas deferens passes over sac and was probably at one time closely attached to its walls.

T, testicles; $\mathrm{E}$, epididymis; V, vas deferens; s, hernial sac; B, band with spermatic artery upon it; s. M. Cut edge of serous membrane. ${ }^{1}$

since it is present in this case of infantile hernia (Fig. 4), it might be supposed to have. something to do with its

' I am indebted to the kindness of Mr. E. Owen and Dr. Silcock for per. mission to examine and draw this specimen. 
formation. Before accepting this inference the absence of the plica vascularis in the case of congenital cæcocele already mentioned (p. 505) entails caution and suggests that the fold, in itself, need not be an essential cause. However, it may be remembered that in its place a quantity of muscular fibres and fibro-areolar tissue passed upon the posterior wall of the hernial sac to the cæcum and probably performed the rôle which, in other cases, has been assigned to the plica vascularis. The pertinence of these remarks will be clearer as the peculiarities of certain cases of infantile hernia are investigated. In the two specimens which are depicted in Figs. 5 and 8 , the plica vascularis is not apparent, having, I think, been removed; but, in its place, a strong fasciculated band extends from the epididymis to the lower extremity of the hernial sac. The drawings show that the spermatic artery is intimately associated with this structure, which is proved by the microscope to consist of unstriped muscle-fibres and fibroareolar tissue. In the St. Mary's specimen the origin of the muscular fasciculi may be traced far down the back of the epididymis, possibly to the scrotum, and in either case they terminated above upon the inner wall of the hernial sac, many of them ascending between the two layers as far as its neck. If the relations of these muscular fibres to the epididymis, spermatic vessels and serous membrane be compared with those which have already been attributed to the upward prolongation of the gubernaculum testis the likeness is manifest, and without doubt they are identical structures. The moment it has been admitted that the band of muscle-fibres, which extends from the epididymis to the sac of these infantile herniæ, is part of the gubernaculum, an explanation of the pathology of that disease is possible. I have already endeavoured to prove that the muscle in question has a most important influence in producing the processus vaginalis and in drawing down the peritoneum, and, if this has been allowed, there can be little difficulty in conceiving that it may, under certain eircumstances; produce an additional sac. Before adduc- 
ing evidence to support this proposition a circumstance which is common to congenital and infantile hernia may be commented upon. Under ordinary conditions the processus vaginalis, after it has served for the transition of the testicle, ceases to grow and develop except at its lowest part; which, stimulated by the presence of the testis, becomes larger and thicker. However, it occasionally happens that the processus vaginalis, instead of undergoing those retrograde atrophic changes, grows and develops, and its lumen, instead of ceasing to exist, increases. When this happens the enlargement is not confined to the serous membrane alone, but, as specimens in the Dupuytren museum show, its blood-vessels, and in all probability other structures, participate. In this way the persistence of the plica vascularis in congenital hernia, being part of a general effect, may be explained; and it is not unlikely that the hypertrophied condition of the upper part of the gubernaculum (internal cremaster) in cases of infantile hernia is related to it. Long ago Cloquet put it upon record that the gubernaculum could create ${ }^{1}$ by its traction the sac of an ordinary hernia and Sir William Lawrence ${ }^{2}$ testifies to the importance of this observation by quoting it in extenso. If this be so, there is no difficulty in believing that the gubernaculum assists in the production of the sac of an infantile hernia. The morbid anatomy of the disease points strongly to the probability of this assumption. The portion of peritoneum from which the sac is formed, that which lines the back of the abdomen, has already been shown to be loose and easily displaced, and, moreover, it has been affirmed that normally the gubernaculum is inserted into it. Therefore, from an anatomical point of view, the idea is tenable. But before accepting this conclusion, that the sac of infantile herniæ is caused by the traction of the gubernaculum testis, the specimens themselves ought to be examined to see whether they lend any support to it. The following points may be noted: $A$, that the sac is

1 Cloquet, ' Causes, \&c., des Hernies,' p. 23, et seq.

2 Lawrence on 'Hernia,' p. 19, et seq. 
always closely related to the posterior wall of the open processus vaginalis and usually bulges into it; $\mathrm{B}$, that the sac is formed from the loose and yielding peritoneum of the back of the abdomen; c, that a band of muscular fibres closely connected with the spermatic vessels is inserted into the inferior extremity and surface of their sac wall. Although these are cogent reasons yet it might be anticipated that a sac, which owes its birth to tractive force, would betray its origin by its conical shape. None of the infantile herniø which have been mentioned are particularly pointed. In one case (Guy's 2497, Fig. 5, p. 495) the posterior wall of the hernial sac exhibits a suggestive pouch which descends behind, and parallel to, the main sac, but in other respects their shape is very like that ordinarily produced by pressure from within. These facts do not forbid the supposition that at the commencement these sacs may not have been originated by the gubernaculum and afterwards modified by pressure, and a specimen which I have dissected countenances this view (Specimen 2140 $0^{\mathrm{B}}$, St. Bartholomew's Hospital Museum). In it the processus vaginalis was represented by a long tube which extends from the internal abdominal ring to just above the epididymis. This tube communicates with the general cavity of the peritoneum by a small aperture, a quarter of an inch in diameter, which occupies the usual position of theinternal abdominal ring external to the epigastric artery. A probe introduced into this opening showed that the processus vaginalis was occluded an inch from its upper end, but in the remainder of its extent its cavity was almost half an inch in diameter, above, and one and a half below. Behind the superior part of this serous tube a hernial sac protruded from the peritoneum in such a way that its anterior wall bulged slightly into the cavity of the processus vaginalis. Attached to the lower extremity of this protrusion and to its posterior wall were strong bands of unstriped muscle-fibre intimately related to the spermatic vessels. The end of the sac to which these were attached was conical and sharply pointed.

VOL. LXIX. 
It seems hardly requisite to enumerate the reasons why this case should be included in the category of infantile hernia, and it clearly shows by its shape that the hernial sac was caused by the traction of the gubernaculum testis. This specimen also demonstrates that in infantile hernia the processus vaginalis need not necessarily communicate with the cavity of the tunica vaginalis, because in it the latter was shut off from the former, in the same way as in funicular hernia. This fact seems also to be displayed by other specimens of infantile hernia in the museum of St. Bartholomew's Hospital (Sp. 2140c and 2140a), but as I have not yet dissected these no other assertions will be made concerning them.

The conclusion arrived at, after studying these data, is that the sac of an infantile hernia owes its origin to the action of the gubernaculum testis, but that afterwards it may be considerably modified by pressure from within. In this way may be explained a circumstance which seems to militate against many of the previous assertions. A glance at Fig. 6, p. 498 shows that the sac of this hernia protrudes in the open processus vaginalis like a cyst pendent] from the ring and that there is no trace of muscular fibres reaching from its extremity to the epididymis. However, when this beautiful specimen is viewed in profile it is clear that its attachment to the posterior wall of the vaginal process is quite an inch long, and although no musclefibres are attached to the bottom of the sac, numerous bands may be perceived running upwards behind the serous membrane (vaginal process), and when they arrive at the fornix, which is formed by the junction of the vaginal process with the outer layer of the hernial sac, they turn forwards and insinuate themselves between the two layers of serous membrane which constitute its walls. The construction of the sac of this hernia, and the condition of the peritoneum at its neck have already been discussed, $v . a$., and it has been decided that it belongs to the infantile variety. This being the case, although it is evident that pressure from within has profoundly affected its 
sac, yet it cannot be denied but that it may have had something to do with its beginning.

Before concluding these observations reference may be made to a point which has not yet been touched upon. In nearly all the cases of infantile hernia which have been mentioned the upper edge of the hernial sac is formed by the posterior margin of the aperture by which the processus vaginalis communicates with the cavity of the peritoneum. It seems natural to ask by what means this acquires its immobility. In the St. Mary's specimen this portion of serous membrane is exceedingly thick and strong, and attached by a species of alæ to the peritoneum of the front wall of the abdomen, which has been shown to be comparatively immobile. In the specimen in the museum of St. Bartholomew's Hospital which has just been described a not dissimilar condition exists, and besides the serous membrane exhibits many old scars and thickenings; but, at the present, it would be premature to express any definite opinions upon this point, for there is reason to think that the neck of the sac may sometimes be produced in a different manner. In conclusion, perhaps, I may be permitted to recapitulate the results arrived at by this inquiry :

$a$. That the London museums contain no specimen of encysted hernia such as is usually described.

$b$. That the various specimens designated by that name belong to the infantile variety.

c. That the latter owe their origin to the tractive power of the gabernaculum testis.

(For a report of the discussion on this paper, see 'Proceedings of the Royal Medical and Chirurgical Society,' New Series, vol. ii, p. 118.) 